\title{
MAGNETO-OPTICAL CHARACTERIZATION OF FeAU ARTIFICIALLY ORDERED ALLOY
}

\author{
Katsuaki Sato, Junya Abe, Hiroshi Ikekame, Koki Takanashi*, Seiji Mitani* \\ and Hiroyasu Fujimori* \\ Tokyo University of Agriculture and Technology, Koganei, Tokyo 184, Japan \\ *Institute for Materials Research, Tohoku University, Katahira, Sendai 980-77, Japan
}

Abstract: Superlattices consisting of $\mathrm{Fe}$ and Au ultrathin layers with thickness of a few monolayers were prepared. Formation of $\mathrm{Ll}_{0}$-type structure in the $\mathrm{Fe}(1 \mathrm{ML}) / \mathrm{Au}(1 \mathrm{ML})$ superlattice was confirmed by $\mathrm{X}$-ray diffraction. Magneto-optical studies suggest formation of a new energy band structure characteristic of the new alloy.

KEYWORDS: Fe/Au multilayers, artificially ordered alloy, magneto-optical polar Kerr effect, spectrum

\section{INTRODUCTION}

Recent development of epitaxial growth technology has made it possible to fabricate artificial structures on an atomic scale.[1] In particular, artificial structures (superlattices or sandwiches) consisting of magnetic and nonmagnetic thin layers have been attracting interest as new functional materials with novel physical properties such as giant magnetoresistance (GMR), large surface magnetic anisotropy and peculiar magneto-optical response characteristic of quantum confinement.[2]

In most of the studies, the physical properties of the layers in the artificial structures, no matter whether they are magnetic or nonmagnetic, have been presumed to keep the same crystal and electronic structures in multilayers as those in the bulk. Only a few investigations have been carried out from the view point that the artificial structure shows completely different electronic structure from those of its constituent layers in their freestanding states.

Recently we succeeded in the artificial preparation of a novel ordered alloy with $L 1_{0}$ crystal structure by means of alternate deposition of $\mathrm{Fe}$ and $\mathrm{Au}$ layers with monoatomic thickness on a $\mathrm{Au}(001)$ buffer layer grown on a $\mathrm{MgO}$ (001) substrate. [3,4] Atomic arrangement in a unit cell of the tetragonal $\mathrm{Ll}_{0}$ ordered structure is given in Fig. 1. Fabrication of such "ordered alloy" structure is quite remarkable, since the Fe-Au system is known to show a peritectic-type phase diagram, which precludes existence of neither intermetallics nor intermediate alloys.

In order to clarify whether the electronic structure of the artificially ordered alloy is that expected from a simple stack of two kinds of material or a completely new one, we measured magneto-optical spectra on the artificial alloy. We found that the magneto-optical spectrum of the artificial $\mathrm{Fe}-\mathrm{Au}$ alloy shows a distinguishing feature which is not found in a Fe film or in a $\mathrm{Fe} / \mathrm{Au}$ multilayer with thick layers. [5]

In the present study we prepared superlattices consisting of $N$ periods of $n$ monoatomic layers (ML) of $\mathrm{Fe}$ and $\mathrm{Au}$ (hereafter denoted as $\left.[\mathrm{Fe}(n \mathrm{ML}) / \mathrm{Au}(n \mathrm{ML})]_{N}\right)$ on $\mathrm{Au}(001) / \mathrm{MgO}(001)$ as well as on $\mathrm{Au}(111) / \mathrm{Al}_{2} \mathrm{O}_{3}$ (11.0) substrates and measured magneto-optical Kerr spectra in these films.

\section{EXPERIMENTAL}

The specimens used in the present study have been prepared by an ultra high vacuum (UHV) deposition technique on either $\mathrm{MgO}(100)$ or $\mathrm{Al}_{2} \mathrm{O}_{3}\left(11^{\circ} 0\right)$ substrates. The base pressure of the deposition system was $3 \times 10^{-10}$ Torr.

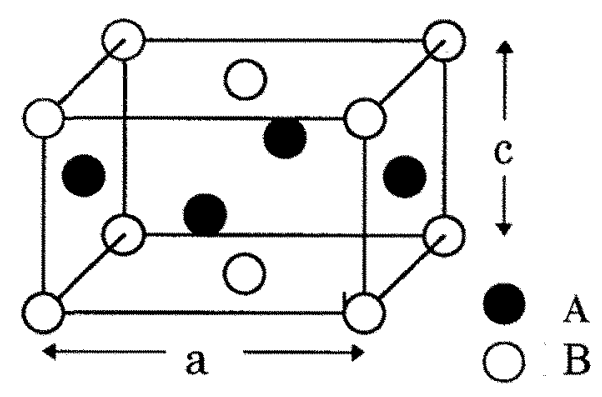

Fig.1 Tetragonal $\mathrm{L}_{0}$ ordered structure 
A Fe seed layer of $1 \mathrm{~nm}$ followed by a $\mathrm{Au}$ buffer layer of $50 \mathrm{~nm}$ was deposited at $200^{\circ} \mathrm{C}$ and subsequently annealed for $30 \mathrm{~min}$ to $1 \mathrm{~h}$ at $500^{\circ} \mathrm{C}$. The orientation of the Au buffer layer was (001) for $\mathrm{MgO}$ substrates and (111) for $\mathrm{Al}_{2} \mathrm{O}_{3}$. The $\mathrm{Fe}$ seed was necessary to control the orientation of the Au layer. Multilayers with $N$ periods, each of which consists of $n$ ML Fe layers and $n$ ML Au layers, were deposited in the UHV system at $70^{\circ} \mathrm{C}$ on the $\mathrm{Au}$ buffer. The orientation of the film surface in $\mathrm{Fe}$ on $\mathrm{Au}(100)$ was (001), while that on $\mathrm{Au}(111)$ was (110). The deposition rates were approximately $0.01 \mathrm{~nm} / \mathrm{s}$. The layer thickness was controlled by using a quartz thickness monitor. Details of preparation techniques were described elsewhere. $[3,4,6]$ Epitaxial growth of $\mathrm{Fe} / \mathrm{Au}$ multilayers were confirmed by RHEED patterns for both series of samples.

These films were characterized by X-ray diffraction (XRD). The $[\mathrm{Fe}(1 \mathrm{ML}) / \mathrm{Au}(1 \mathrm{ML})]_{100}$ multi-layer on $A u(001)$ clearly showed a diffraction line $\left(2 \theta=22.9^{\circ}\right)$ characteristic of the tetrahedral $\mathrm{Ll}_{0}$ ordered structure. For $\mathrm{Fe} / \mathrm{Au}$ multilayers with thicker layers, i.e., $[\mathrm{Fe}(n \mathrm{ML}) / \mathrm{Au}(n \mathrm{ML})]_{N}(n=2-5)$, satellite lines due to multilayered structure were clearly observed.

On the other hand, no such diffraction lines were observed in the $[\mathrm{Fe}(1 \mathrm{ML}) / \mathrm{Au}(1 \mathrm{ML})]_{100}$ film on $\mathrm{Au}$ (111). This fact rules out formation of any ordered structure in this multilayer. However, in $[\mathrm{Fe}(n \mathrm{ML}) / \mathrm{Au}(n \mathrm{ML})]_{N}(n=2$ and 3) on $\mathrm{Au}(111)$, well-defined superlattice lines of $1 \mathrm{st}, 3 \mathrm{rd}$ and 5 th order were observed, indicating the formation of a superstructure.

Table I
Structure parameters of
\begin{tabular}{|l|c|c|c|c|}
\hline $\begin{array}{c}\text { Au buffer } \\
\text { orientation }\end{array}$ & $\mathrm{n}$ & $\mathrm{NML}) / \mathrm{Au}(n \mathrm{ML})]_{N}$ multilayers \\
\hline Aur(001) & 1 & $\begin{array}{c}\mathrm{N} \\
\text { (periods) }\end{array}$ & $\begin{array}{c}\text { thickness for } \\
\text { one period (nm) }\end{array}$ & $\begin{array}{c}\text { average } \\
\text { spacing (nm) }\end{array}$ \\
& 2 & 100 & 0.3800 & 0.190 \\
& 3 & 30 & 0.7272 & 0.1818 \\
& 4 & 25 & 1.0744 & 0.1791 \\
& 5 & 20 & 1.4216 & 0.1777 \\
& 10 & 10 & 5.600 & 0.1769 \\
& 1 & 100 & 0.448 & 0.2800 \\
\hline Au(111) & 2 & 50 & 0.892 & 0.224 \\
& 3 & 33 & 1.344 & 0.223 \\
& 2 & 33 & 0.224 \\
\hline
\end{tabular}

The designed layer thickness, the number of periods and the average lattice-plane spacing normal to the film plane obtained by XRD are listed in Table 1.

Magneto-optical Kerr rotation and ellipticity were measured at room temperature between 1.2 and $6 \mathrm{eV}$ using a specially designed Kerr spectrometer.[7] The maximum field applied was $1.7 \mathrm{~T}$. Optical reflectivity was measured using a Hitachi U-3410 spectrophotometer, from which optical constants were deduced by the KramersKronig analysis with the help of the data values measured by a Woollam type WVASE spectroscopic ellipsometer between 2 and $5 \mathrm{eV}$.

\section{RESULTS AND DISCUSSION}

\section{Fe/Au MULTILAYERS ON Au(001)}

Figure 2 illustrates spectra of polar magnetooptical Kerr rotation $\theta_{\mathrm{K}}$ (solid line) and Kerr ellipticity $\eta_{\mathrm{K}}$ (dotted line) in the $[\mathrm{Fe}(10 \mathrm{ML})$ $/ \mathrm{Au}(10 \mathrm{ML})]_{10}$ multilayer on $\mathrm{Au}(001)$ buffer, which shows a prominent peak of Kerr rotation $\theta_{\mathrm{K}}$ and a well-defined dispersion-type Kerr ellipticity $\eta_{\mathrm{K}}$ at $2.4 \mathrm{eV}$. This structure may be caused by the enhancement of $\mathrm{MO}$ signal by rapid change of optical constants in the Au layers as simulated by means of virtual optical constant method described in the preceding paper.[5]

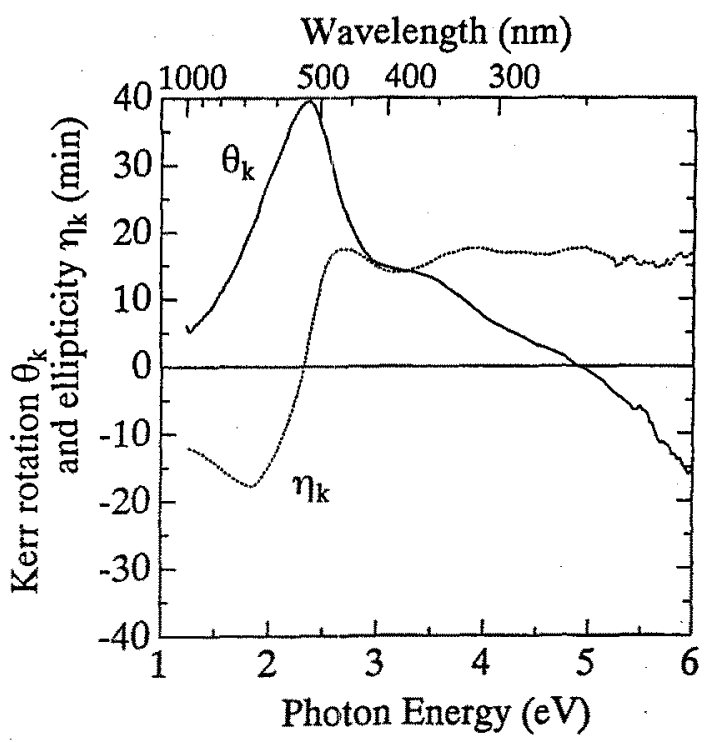

Fig. 2 Spectra of magneto-optical Kerr rotation $\theta_{\mathrm{K}}$ (solid curve) and Kerr ellipticity $\eta_{K}$ (dotted curve) in $[\mathrm{Fe}(10 \mathrm{ML}) / \mathrm{Au}(10 \mathrm{ML})]_{10}$ multilayers on $\mathrm{Au}(001)$ buffer 


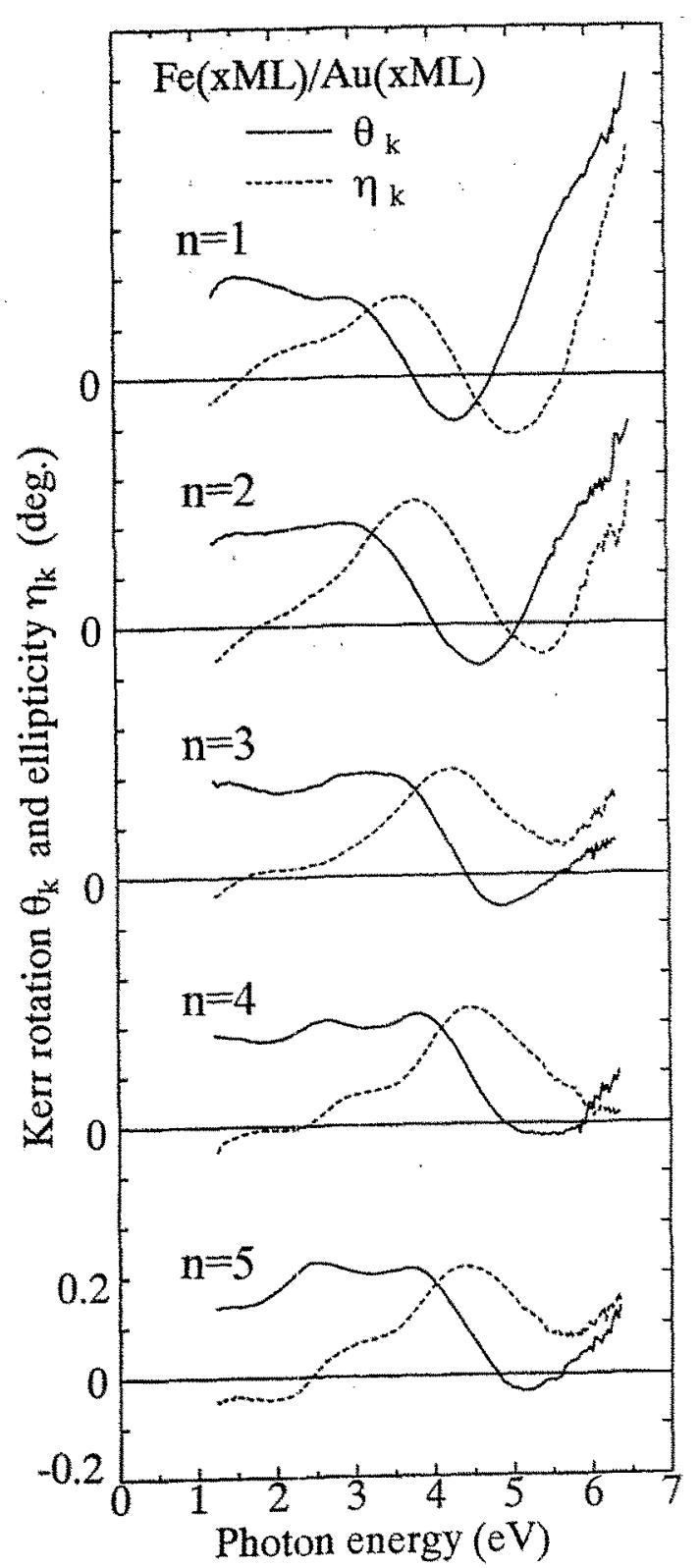

Fig. 3 Spectra of magneto-optical Kerr rotation $\theta_{\mathrm{K}}$ (solid curve) and Kerr ellipticity $\eta_{\mathrm{K}}$ (dotted curve) in $\mathrm{Fe}(\mathrm{nML}) / \mathrm{Au}(\mathrm{nML})(1 \leq n \leq 5)$ multilayers on $\mathrm{Au}(001)$ buffer.

Magneto-optical Kerr spectra in [Fe(nML) $/ \mathrm{Au}(n \mathrm{ML})]_{N}$ films with thin $\mathrm{Fe}$ and $\mathrm{Au}$ layers $(1 \leq n \leq 5)$ prepared on $\mathrm{Au}(001)$ are shown in Fig. 3. The spectral features of these multilayers are completely different from that of $n=10$. No clear enhancement effect was observed around $2.4 \mathrm{eV}$. The peak values of rotation below $4 \mathrm{eV}$ do not exhibit a drastic change with layer thickness. A prominent dispersion-type structure centered at $3.6 \mathrm{eV}$ is observed in the Kerr rotation spectrum for $n=1$, which undergoes a systematic "blue shift" as $n$ is increased. The amount of the energy shift between $n=1$ and $n=5$ samples is approximately 1 $\mathrm{eV}$.

Comparison between MO spectra of the sample with $n=10$ and those with $n=1-5$ leads to a conclusion that the electronic structures of the $\mathrm{Au}$ layer in the $\mathrm{Fe} / \mathrm{Au}$ multilayers deposited on $\mathrm{Au}(001)$ with $n=1-5$ are not similar to those of thick Au layers which may be assumed to hold the bulk nature.

In order to clarify whether or not the magnetooptical structures observed around $4 \mathrm{eV}$ in Fig. 3 are related to the plasma frequecies, the reflectivity spectra were measured between 0.5 and $7 \mathrm{eV}$. Some examples of experimental results are illustrated in Fig. 4. The reflectivity spectrum in the $\mathrm{Fe}(10 \mathrm{ML}) / \mathrm{Au}(10 \mathrm{ML})$ multilayer shows a distinct break point around $2.4 \mathrm{eV}$, where the peak of Kerr rotation occurs. On the other hand, the reflectivity undergoes a monotonous decrease up to the highest energy of the measurement in multilayers consisting of thin layers of $\mathrm{Fe}$ and $\mathrm{Au}(n=1-5)$.

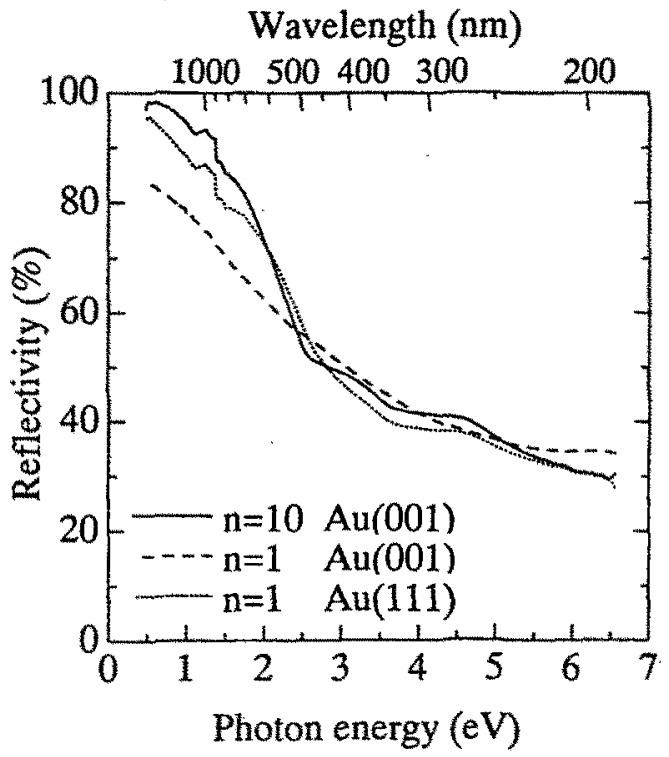

Fig. 4 Typical examples of relectivity spectra of Fe/Au multilayers. Solid curve represents $[\mathrm{Fe}(10 \mathrm{ML}) / \mathrm{Au}(10 \mathrm{ML})]_{10}$ multilayers on $\mathrm{Au}(001)$, dashed curve $[\mathrm{Fe}(1 \mathrm{ML}) / \mathrm{Au}(1 \mathrm{ML})]_{100}$ on $\mathrm{Au}(001)$ and dotted curve [Fe(1ML)/Au(IML) $]_{100}$ on Au (111).

Real and imaginary parts of the dielectric function were calculated by the Kramers-Kronig analysis of the reflectivity. We used the optical constants (for photon energies between 1.5 and 3 
eV) measured by means of spectroscopic ellipsometry to calibrate the values of the dielectric constants.

As a typical example, real and imaginary parts of the diagonal component of dielectric function in $[\mathrm{Fe}(1 \mathrm{ML}) / \mathrm{Au}(1 \mathrm{ML})]_{100}$ are shown in Fig. 5. No drastic change of spectral features were observed in $n \geq 2$ multilayers. As shown in Fig. 5 real part of diagonal dielectric function $\varepsilon_{x x}$ never crosses the abscissa near the photon energy ( 3.6 $\mathrm{eV})$ corresponding to the peak of Kerr rotation. Thus it is found that the Kerr rotation peak cannot be ascribed to enhancement by plasma resonance.

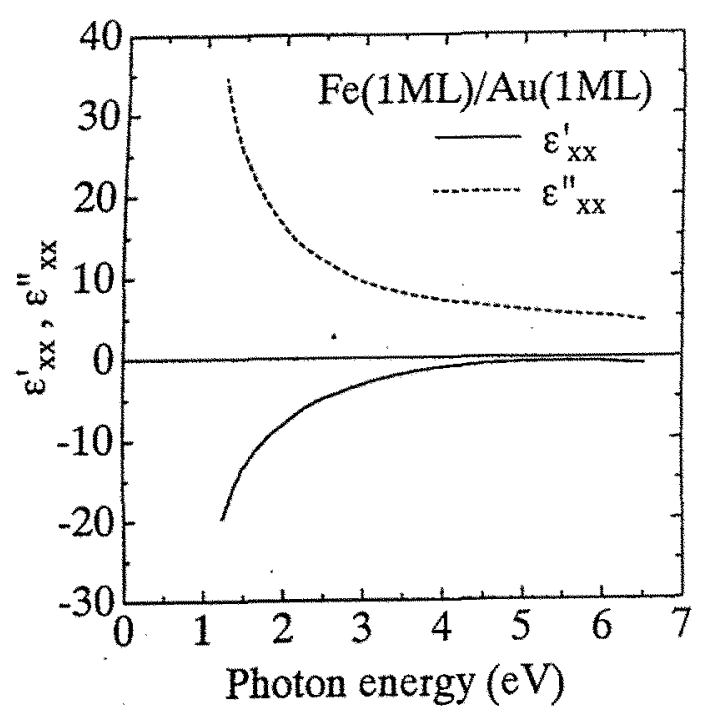

Fig. 5 Spectrum of dielectric function in [Fe(1ML)/Au(1ML)] on Au (001) buffer.

From experimentally obtained data of $\theta_{\mathrm{K}}, \eta_{\mathrm{K}}$ and $\varepsilon_{\mathrm{xx}}$ we evaluated off-diagonal elements of conductivity multiplied by angular frequency $\omega \sigma_{\mathrm{xy}}$, which is known to be useful for discussion of magnetooptical effect in terms of the electronic structures. The spectra are given in Fig. 6.

It should be noted that $\omega \sigma^{\prime \prime}{ }_{x y}$ takes an approximately constant value $\left(\sim-2 \times 10^{29} \mathrm{~s}^{-2}\right)$ between 1 and $4 \mathrm{eV}$. Erskine and Stern argued that a constant value of $\omega \sigma^{\prime \prime}{ }_{x y}$ can be correlated with the spin polarization of conduction electrons. [8] Such behavior has not been observed in the $\omega \sigma^{\prime \prime}{ }_{\text {xy }}$ spectrum of $\mathrm{Fe}$ and suggests that electronic states at the Fermi surface in the $\mathrm{Fe} / \mathrm{Au}$ multilayers are completely different from those of Fe.
On the constant value, a peak of the off-diagonal conductivity is superposed around $4-5 \mathrm{eV}$. We believe that the $4 \mathrm{eV}$ transition originate from band-to-band transition in the new energy band scheme of the superlattice.

To confirm this point we made a rough estimate of $\omega \sigma^{\prime \prime}{ }_{x y}$ using the density of states (DOS) curve obtained by the electronic band structure calculations in the Fe[1ML] $/ \mathrm{Au}[1 \mathrm{ML}]$ superlattice. [9]

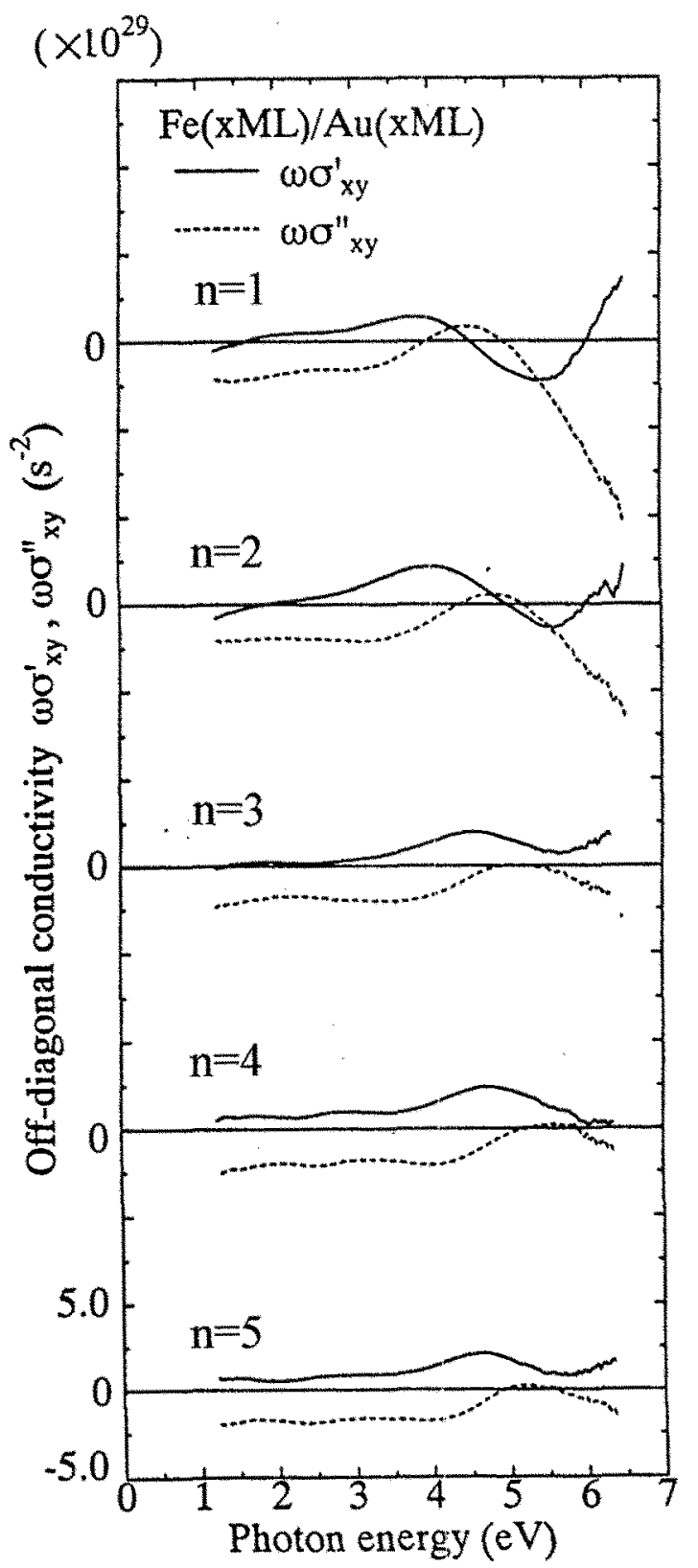

Fig. 6 Spectra of $\omega \sigma^{\prime \prime}{ }_{x y}$ in $[\mathrm{Fe}(n \mathrm{ML}) / \mathrm{Au}(n \mathrm{ML})]_{N}$ multilayers on $\mathrm{Au}(001)$. 
The spectrum of $\omega \sigma^{\prime \prime}{ }_{x y}$ was evaluated by taking an energy-derivative of the joint density of states derived from a convolution between the filled and the empty states in the DOS curves, the same procedure having been described elsewhere.[10] The calculated spectrum of $\omega \sigma^{\prime \prime}{ }_{x y}$ is shown in Fig. 7. A distinct peak is found around $4 \mathrm{eV}$ in the spectrum, which arises mainly from the filled $5 \mathrm{~d}$ states (down spin) of Au to the empty $3 \mathrm{~d}$ states (down spin) of Fe. This result supports the idea that the Fe[1ML]/Au[1ML] superlattice is not a simple stack of $\mathrm{Fe}$ and $\mathrm{Au}$ layers but a novel ordered alloy between them.

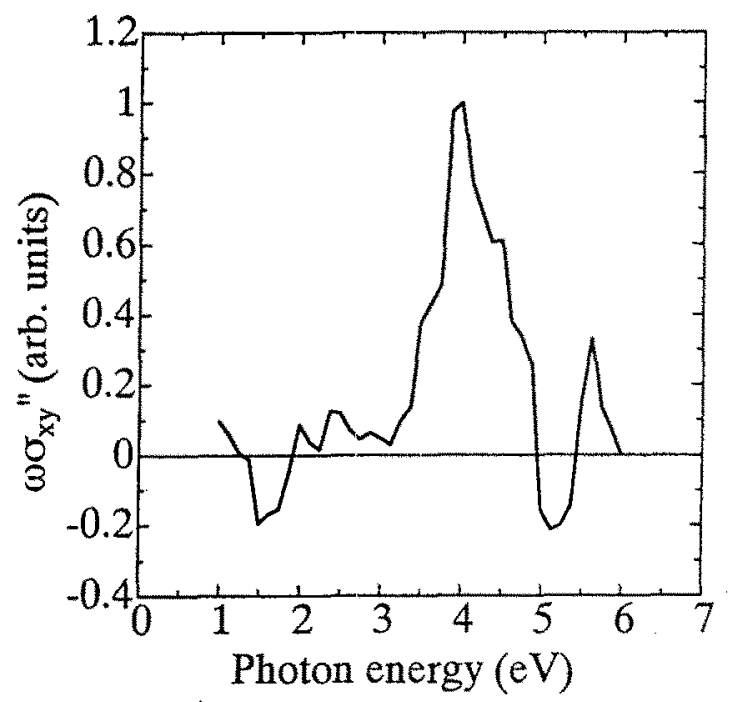

Fig. 7 A spectrum of $\omega \sigma^{\prime \prime} x y$ estimated from the density of state curve.

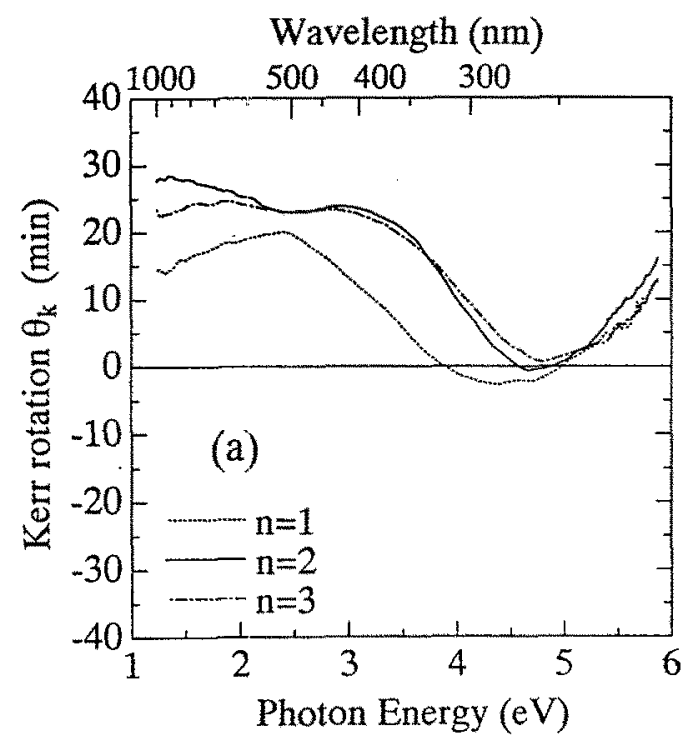

We simulated spectra for $n \geq 2$ by the virtual optical constant method assuming that the superlattice consists of interfacial $n=1$ alloy and "bulk" layers of Fe and Au. The calculated spectrum showed a peak at the plasma edge of $\mathrm{Au}$, while the characteristic dip remained at the same position although the absolute value of rotation is smaller.

\section{Fe/Au MULTILAYERS ON Au (111)}

Figures 8(a) and 8(b) illustrates magnetooptical Kerr rotation and Kerr ellipticity in $[\mathrm{Fe}(n \mathrm{ML}) / \mathrm{Au}(n \mathrm{ML})]_{N}$ multilayer with $n=1,2$ and 3 on $\mathrm{Au}(111)$. The absolute values of peak Kerr rotation of these multilayers on $\mathrm{Au}$ (111) are approximately twice as large as that of multilayers deposited on $\mathrm{Au}(001)$ buffer.

It should also be noted that the multilayer with $n=1$ shows different $\mathrm{MO}$ spectra from the rest. The spectral feature of the former is quite similar to that of $n=10$ multilayer on $\mathrm{Au}(001)$, with a broad peak of Kerr rotation around $2.5 \mathrm{eV}$, although the absolute value of rotation is smaller.

On the other hand, spectra of $[\mathrm{Fe}(n \mathrm{ML})$ $/ \mathrm{Au}(n \mathrm{ML})]_{N}$ multilayer on $\mathrm{Au}(111)$ for $n=2$ and 3 resemble with each other. They both show striking similarity with the $\mathrm{MO}$ spectrum of $\mathrm{Fe} / \mathrm{Au}$ with $n=3$ on $\mathrm{Au}(001)$. This suggests the electronic structures of periodic multilayers on $\mathrm{Au}$ (111) are not so much different from those on $\mathrm{Au}(001)$.



Fig. 8 Spectra of magneto-optical Kerr rotation (a)and Kerr ellipticity (b) in $[\mathrm{Fe}(n \mathrm{ML}) / \mathrm{Au}(n \mathrm{ML})]_{v}$ on $\mathrm{Au}(111)$ for $\mathrm{n}=1$ (dotted), 2 (solid) and 3 (dot-dashed). 
As was shown in Fig. 4 , the reflectivity spectrum of the $n=1$ multilayer on $\mathrm{Au}(111)$ is quite similar to that of the $n=10$ film on $\mathrm{Au}(001)$, but is different from the $n=1$ multilayer on $\mathrm{Au}(001)$.

As described earlier, the $n=1$ multilayer on $\mathrm{Au}(111)$ does not show any superlattice line in XRD pattern, whereas multilayers with $n=2,3$ show a periodic structure. Therefore it may be postulated from these experiments that the electronic structures of multilayers are different from those of a simple stack of two constituent layers as far as an artificial ordered structure is formed.

Lack of periodic structure in the $n=1$ multilayer may be attributed to inferior surface flatness of the Au(111) surface, for which an improvement of the growth technique should be saught in future studies.

\section{CONCLUSION}

From the optical and magneto-optical spectra it has been elucidated that the electronic structures of the $[\mathrm{Fe}(n \mathrm{ML}) / \mathrm{Au}(n \mathrm{ML})]_{N}$ multilayer with $n=1$ 5 are completely different from that of a simple stack of Fe and Au layers. The existence of spinpolarized conduction electrons at the Fermi surface is suggested from the spectra of offdiagonal conductivity. Magneto-optical structure around $4 \mathrm{eV}$ was attributed to an optical transition between $\mathrm{Au} 5 \mathrm{~d}$ and $\mathrm{Fe} 3 \mathrm{~d}$ states.

\section{REFERENCES}

[1] As a general review, "Ultrathin Magnetic Structures", eds. J.A.C. Bland and B. Heinrich, Springer, Berlin, 1994

[2] Y. Suzuki, T. Katayama, W. Geerts, P. Bruno and $\mathrm{H}$. Sawada, Mat. Res. Soc. Symp. Proc. 382, 237-246, 1995

[3] K. Takanashi, S. Mitani, M. Sano, H. Fujimori, H. Nakajima and A. Osawa: Appl. Phys. Lett. 67, 1016-1018 (1995)

[4] S. Mitani, K. Takanashi, H. Nakajima, K. Sato, R. Schreiber, P. Grünberg and H. Fujimori: J. Magn. Magn. Mater. (1996) in press.

[5] K. Sato, T. Kondo, J. Abe, H. Ikekame, M. Sano, K. Takanashi and H. Fujimori: J. Magn. Soc. Jpn. 20, 197-200 (1996)

[6] H. Nakazawa, S. Mitani, K. Takanashi, H. Nakajima, A. Osawa and H. Fujimori: J. Magn. Soc. Jpn. 20, 353-356 (1996) (in Japanese)

[7] K. Sato, H.Hongu, H.lkekame, Y.Tosaka, M.Watanabe, K.Takanashi and H.Fujimori: Jpn. J. Appl. Phys. 32 Part 1 [2] (1993) 989995.

[8] J.L. Erskine and E.A. Stern: Phys. Rev. B8, 1239 (1973)

[9] M.E.McHenry, J.M. MacLaren, M.E. Eberhart and S. Crampin: J. Magn. Magn. Mater. 88, 134-150 (1990)

[10] K. Sato, Y. Aman and H. Hongu: J. Magn. Magn. Mater. 104-107, 1947 (1992). 